JOURNAL OF

SYMPLECTIC GEOMETRY

Volume 6, Number 3, 239-246, 2008

\title{
ON THE SYMPLECTIC FORM OF THE MODULI SPACE OF PROJECTIVE STRUCTURES
}

\author{
Pablo Arés-Gastesi and Indranil Biswas
}

\begin{abstract}
Let $S$ be a $C^{\infty}$ compact connected oriented surface whose genus is at least two. Let $\mathcal{P}(S)$ be the moduli space of isotopic classes of projective structures associated to $S$. The natural holomorphic symplectic form on $\mathcal{P}(S)$ will be denoted by $\Omega_{P}$. The natural holomorphic symplectic form on the holomorphic cotangent bundle $T^{*} \mathcal{T}(S)$ of the Teichmüller space $\mathcal{T}(S)$ associated to $S$ will be denoted by $\Omega_{T}$. Let $e: \mathcal{T}(S) \longrightarrow \mathcal{P}(S)$ be the holomorphic section of the canonical holomorphic projection $\mathcal{P}(S) \longrightarrow \mathcal{T}(S)$, given by the Earle uniformization. Let $T_{e}: T^{*} \mathcal{T}(S) \longrightarrow \mathcal{P}(S)$ be the biholomorphism constructed using the section $e$. We prove that $T_{e}^{*} \Omega_{P}=\pi \cdot \Omega_{T}$. This remains true if $e$ is replaced by a large class of sections that include the one given by the Schottky uniformization.
\end{abstract}

\section{Introduction}

A projective structure on a smooth compact connected oriented surface $S$ is defined by giving a covering of $S$ by coordinate charts, where the coordinate functions are orientation preserving diffeomorphisms to open subsets of $\mathbb{C}$, such that all the transition functions are Möbius transformations. Two projective structures are called equivalent if they differ by a diffeomorphism of $S$ homotopic to the identity map. Let $\mathcal{P}(S)$ denote the equivalence classes of projective structures on $S$.

The Teichmüller space $\mathcal{T}(S)$ for $S$ parametrizes all the equivalence classes of complex structures on $S$ compatible with its orientation; two complex structures are called equivalent if they differ by a diffeomorphism of $S$ homotopic to the identity map. Both $\mathcal{P}(S)$ and $\mathcal{T}(S)$ are complex manifolds, 
and $\operatorname{dim} \mathcal{P}(S)=2 \cdot \operatorname{dim} \mathcal{T}(S)$. There is a natural surjective holomorphic submersion

$$
\varphi: \mathcal{P}(S) \longrightarrow \mathcal{T}(S)
$$

that sends a projective structure on $S$ to the underlying complex structure on $S$.

The above projection $\varphi$ makes $\mathcal{P}(S)$ a torsor over $\mathcal{T}(S)$ for the holomorphic cotangent bundle $T^{*} \mathcal{T}(S)$. This means in particular that the fiber of $\varphi$ over any point $X \in \mathcal{T}(S)$ is an affine space for the space of all holomorphic quadratic differentials on the Riemann surface corresponding to $X$. Consequently, any smooth section

$$
f: \mathcal{T}(S) \longrightarrow \mathcal{P}(S)
$$

of the above projection $\varphi$ produces a diffeomorphism

$$
T_{f}: T^{*} \mathcal{T}(S) \longrightarrow \mathcal{P}(S)
$$

that sends $(X, \omega)$ to the projective structure $f(X)+\omega$, where $X \in \mathcal{T}(S)$ and $\omega$ is a holomorphic quadratic differential on the Riemann surface $X$. If the section $f$ is holomorphic, then $T_{f}$ is a biholomorphism.

Both $T^{*} \mathcal{T}(S)$ and $\mathcal{P}(S)$ are equipped with natural holomorphic symplectic structures. Let $\Omega_{T}$ (respectively, $\Omega_{P}$ ) denote the canonical symplectic form on $T^{*} \mathcal{T}(S)$ (respectively, $\mathcal{P}(S)$ ).

Assume that genus $(S) \geq 2$. We prove the following (Theorem 3.1):

Theorem 1.1. Let

$$
e: \mathcal{T}(S) \longrightarrow \mathcal{P}(S)
$$

be the holomorphic section given by the Earle uniformization. Then

$$
T_{e}^{*} \Omega_{P}=\pi \cdot \Omega_{T},
$$

where $T_{e}: T^{*} \mathcal{T}(S) \longrightarrow \mathcal{P}(S)$ is the biholomorphism given by the section $e$.

Theorem 1.1 extends to sections $f: \mathcal{T}(S) \longrightarrow \mathcal{P}(S)$ as above that satisfy certain conditions (see Remark 3.2). Another example of $f$ with this property is the section given by the Schottky uniformization.

The proof of Theorem 1.1 is based on theorems of S. Kawai and C. T. McMullen.

\section{Symplectic structure on the moduli of projective structures}

Fix a connected compact oriented $C^{\infty}$ surface $S$ of genus $g$ with $g \geq 2$. Let $\mathcal{T}(S)$ denote the Teichmüller space associated to $S$. Therefore,

$$
\mathcal{T}(S)=\operatorname{Conf}(S) / \operatorname{Diff}^{0}(S)
$$

where $\operatorname{Conf}(S)$ is the space of all conformal structures on $S$ compatible with the orientation of $S$, and $\operatorname{Diff}^{0}(S)$ is the group of all diffeomorphisms of $S$ homotopic to the identity map of $S$. The Teichmüller space $\mathcal{T}(S)$ is a 
complex manifold of complex dimension $3 g-3$, and it is diffeomorphic to $\mathbb{R}^{6 g-6}$.

Similarly, we have the moduli space of projective structures associated to $S$. To explain this with more detail, we first recall the definition of a projective structures on $S$.

A projective structure on $S$ is given by data $\left\{U_{i}, \phi_{i}\right\}_{i \in I}$, where

- $U_{i} \subset S$ are open subsets with $\bigcup_{i \in I} U_{i}=S$, and

- $\phi_{i}: U_{i} \longrightarrow \mathbb{C P}^{1}$ are orientation preserving diffeomorphisms from $U_{i}$ to $\phi_{i}\left(U_{i}\right)$ satisfying the condition that for each ordered pair $i, k \in I$, there is some element

$$
G_{i, k} \in \operatorname{PGL}(2, \mathbb{C})=\operatorname{Aut}\left(\mathbb{C P}^{1}\right)
$$

such that the map

$$
\phi_{k} \circ \phi_{i}^{-1}: \phi_{i}\left(U_{i} \cap U_{k}\right) \longrightarrow \phi_{k}\left(U_{i} \cap U_{k}\right)
$$

coincides with the restriction of the automorphism $G_{i, k}$ of $\mathbb{C P}^{1}$.

Two data $\left\{U_{i}, \phi_{i}\right\}_{i \in I}$ and $\left\{U_{j}, \phi_{j}\right\}_{j \in J}$ of the above type are called equivalent if their union $\left\{U_{k}, \phi_{k}\right\}_{k \in I \cup J}$ also satisfies the above conditions. A projective structure on $X$ is an equivalence class of data. (See $[\mathbf{2}]$ for various alternative descriptions of a projective structure.)

Define

$$
\mathcal{P}(S)=\operatorname{Proj}(S) / \operatorname{Diff}^{0}(S),
$$

where $\operatorname{Proj}(S)$ is the space of all projective structures on $S$, and $\operatorname{Diff}^{0}(S)$ is the group in (2.1).

It is known that $\mathcal{P}(S)$ is a complex manifold of complex dimension $6 g-6$, and it is diffeomorphic to $\mathbb{R}^{12 g-12}$. The complex manifold $\mathcal{P}(S)$ has a natural holomorphic symplectic structure. We will briefly recall its description.

A projective structure $P$ on $S$ gives a flat principal $\operatorname{PGL}(2, \mathbb{C})$-bundle over $S$. For any given data $\left\{U_{i}, \phi_{i}\right\}_{i \in I}$ of above type defining $P$, consider the trivial principal $\operatorname{PGL}(2, \mathbb{C})$-bundle $U_{i} \times \operatorname{PGL}(2, \mathbb{C})$ on each $U_{i}$. For any ordered pair $i, k \in I$, these trivial principal $\mathrm{PGL}(2, \mathbb{C})$-bundles on $U_{i}$ and $U_{k}$ may be glued together over $U_{i} \cap U_{k}$ using $G_{i, k} \in \operatorname{PGL}(2, \mathbb{C})$ as the transition function, where $G_{i, k} \in \operatorname{PGL}(2, \mathbb{C})$ is the element giving the map in (2.2). This way we get a flat principal PGL(2, $\mathbb{C})$-bundle over $S$ associated to $P$. Consequently, we get a map

$$
h: \mathcal{P}(S) \longrightarrow \operatorname{Hom}\left(\pi_{1}(S), \operatorname{PGL}(2, \mathbb{C})\right) / \operatorname{PGL}(2, \mathbb{C})
$$

from $\mathcal{P}(S)$ in (2.3) that sends any projective structure to the holonomy of the corresponding flat principal $\operatorname{PGL}(2, \mathbb{C})$-bundle on $S$.

We note that for two different base points $s_{1}$ and $s_{2}$ of $S$, there is an identification of $\pi_{1}\left(S, s_{1}\right)$ with $\pi_{1}\left(S, s_{2}\right)$ unique up to an inner automorphism (by fixing a path connecting $s_{1}$ to $s_{2}$ ). Therefore, the quotient space 
$\operatorname{Hom}\left(\pi_{1}(S), \operatorname{PGL}(2, \mathbb{C})\right) / \operatorname{PGL}(2, \mathbb{C})$ in $(2.4)$ does not depend on the choice of the base point needed to define the fundamental group.

A homomorphism $\rho_{0}: \pi_{1}(S) \longrightarrow \operatorname{PGL}(2, \mathbb{C})$ is called irreducible if the subgroup image $\left(\rho_{0}\right) \subset \operatorname{PGL}(2, \mathbb{C})$ does not fix any point of $\mathbb{C P}^{1}$. Let

$$
\mathcal{R} \subset \operatorname{Hom}\left(\pi_{1}(S), \operatorname{PGL}(2, \mathbb{C})\right) / \operatorname{PGL}(2, \mathbb{C})
$$

be the space of all irreducible representations. This irreducible representation space $\mathcal{R}$ is a complex manifold of complex dimension $6 g-6$ equipped with a holomorphic symplectic structure [4]. The image of the map $h$ in (2.4) lies in $\mathcal{R}$.

The map $h$ is locally a biholomorphism, which means that $h$ is holomorphic, and for each point $P \in \mathcal{P}(S)$, the differential of $h$ at $P$ is an isomorphism of tangent spaces $[\mathbf{6}, \mathbf{7}]$. Therefore, the holomorphic symplectic form on $\mathcal{R}$ pulls back, by $h$, to a holomorphic symplectic form on $\mathcal{P}(S)$. Let

$$
\Omega_{P} \in H^{0}\left(\mathcal{P}(S), \Omega_{\mathcal{P}(S)}^{2}\right)
$$

be the holomorphic symplectic form on $\mathcal{P}(S)$ obtained this way.

There is a natural map from $\mathcal{P}(S)$ to the Teichmüller space

$$
\varphi: \mathcal{P}(S) \longrightarrow \mathcal{T}(S)
$$

that sends any projective structure on $S$ to the underlying complex structure on $S$. It is known that $\varphi$ is a holomorphic surjective submersion. For any $X \in \mathcal{T}(S)$, the fiber $\varphi^{-1}(X)$ is an affine space for the vector space $H^{0}\left(X, K_{X}^{\otimes 2}\right)$ of all holomorphic quadratic differentials on the Riemann surface $X$ (see $[\mathbf{2}, \mathbf{5}, \mathbf{7}]$ for details).

Let $T^{*} \mathcal{T}(S)$ be the total space of the holomorphic cotangent bundle of $\mathcal{T}(S)$. Therefore, the fiber of $T^{*} \mathcal{T}(S)$ over any $X \in \mathcal{T}(S)$ is $H^{0}\left(X, K_{X}^{\otimes 2}\right)$. Take any smooth section

$$
f: \mathcal{T}(S) \longrightarrow \mathcal{P}(S)
$$

of the projection $\varphi$ in (2.6); so $\varphi \circ f=\operatorname{Id}_{\mathcal{T}(S)}$. Using $f$ we have a diffeomorphism

$$
T_{f}: T^{*} \mathcal{T}(S) \longrightarrow \mathcal{P}(S)
$$

that sends any $(X, \omega) \in T^{*} \mathcal{T}(S)$, where $\omega$ is a holomorphic quadratic differential on the Riemann surface $X$, to the projective structure $f(X)+\omega$ on $X$. If $f$ is a holomorphic section, then the diffeomorphism $T_{f}$ is a biholomorphism.

The complex manifold $T^{*} \mathcal{T}(S)$ being the total space of the cotangent bundle of a complex manifold has a canonical holomorphic symplectic structure. To describe this symplectic form, let $\sigma$ be the tautological Liouville one-form on $T^{*} \mathcal{T}(S)$ that sends any tangent vector $v$ at a point $(z, w) \in T^{*} \mathcal{T}(S)$, where $z \in \mathcal{T}(S)$ and $w \in T_{z}^{*} \mathcal{T}(S)$, to $w(d p(v)) \in \mathbb{C}$; here

$$
d p: T T^{*} \mathcal{T}(S) \longrightarrow p^{*} T \mathcal{T}(S)
$$


is the differential of the natural projection

$$
p: T^{*} \mathcal{T}(S) \longrightarrow \mathcal{T}(S) .
$$

The two-form $d \sigma$ defines a holomorphic symplectic structure on $T^{*} \mathcal{T}(S)$. This symplectic form $d \sigma$ on $T^{*} \mathcal{T}(S)$ will also be denoted by $\Omega_{T}$.

For particular choices of the section $f$ in (2.7) we may ask whether the diffeomorphism $T_{f}$ in (2.8) takes the symplectic form $\Omega_{T}$ on $T^{*} \mathcal{T}(S)$ defined above to a constant multiple of the symplectic form $\Omega_{P}$ constructed in (2.5).

If we fix a base point $X_{0} \in \mathcal{T}(S)$, there a section

$$
B:=B_{X_{0}}: \mathcal{T}(S) \longrightarrow \mathcal{P}(S)
$$

constructed by Bers using the notion of simultaneous uniformization. More precisely, for any $X \in \mathcal{T}(S)$, the projective structure $B_{X_{0}}(X)$ is given by the quasifuchsian group that uniformizes $X$ and $\bar{X}_{0}$, where $\bar{X}_{0}$ is the quotient of the lower half plane by the Fuchsian group for $X_{0}$ (see [1]). In [8], Kawai showed that when $f$ in (2.7) is the section $B$ in (2.9), then

$$
T_{B}^{*} \Omega_{P}=\pi \cdot \Omega_{T},
$$

where $T_{B}$ is constructed as in $(2.8)$ (see $[\mathbf{8}$, p. 165 , Theorem]).

\section{Earle uniformization}

In [3], Earle constructed a canonical holomorphic section

$$
e: \mathcal{T}(S) \longrightarrow \mathcal{P}(S)
$$

The section $e$ depends on the marked surface defined by the fixed surface $S$ as well as on the choice of an involution of the fundamental group of $S$ induced by some orientation reversing diffeomorphism of $S$. In particular, unlike the section $B$ in (2.9) constructed by Bers, the section $e$ does not require fixing a base point of $\mathcal{T}(S)$ for its definition. In this sense, this section $e$ is intrinsic (see the first paragraph of [3, p. 527]). It should be clarified that this section $e$ is not equivariant for the natural actions of the mapping class group $\operatorname{Diff}_{+}(S) / \operatorname{Diff}^{0}(S)$ on $\mathcal{T}(S)$ and $\mathcal{P}(S)$ (here $\operatorname{Diff}_{+}(S)$ is the group of orientation preserving diffeomorphisms of $S$ ). Let

$$
T_{e}: T^{*} \mathcal{T}(S) \longrightarrow \mathcal{P}(S)
$$

be the biholomorphism constructed as in (2.8) from the section $e$ in (3.1).

Theorem 3.1. For the biholomorphism $T_{e}$ in (3.2),

$$
T_{e}^{*} \Omega_{P}=\pi \cdot \Omega_{T},
$$

where $\Omega_{P}$ and $\Omega_{T}$ are the natural holomorphic symplectic forms on $\mathcal{P}(S)$ and $T^{*} \mathcal{T}(S)$, respectively. 
Proof. Let

$$
\theta:=e-B \in C^{\infty}\left(\mathcal{T}(S), T^{*} \mathcal{T}(S)\right)
$$

be the smooth $(1,0)$-form on $\mathcal{T}(S)$, where $e$ and $B$ are the sections in (3.1) and (2.9) respectively. Recall that the space of projective structures on a given Riemann surface compatible with its complex structure is an affine space for the space of all holomorphic quadratic differentials on it, which implies that $e-B$ is a $(1,0)$-form on $\mathcal{T}(S)$. We will first show the following.

For the biholomorphism $T_{e}$ in (3.2),

$$
T_{e}^{*} \Omega_{P}=\pi \cdot \Omega_{T}
$$

if and only if

$$
d \theta=0
$$

where $\theta$ is constructed in (3.3).

To prove this, let

$$
F_{\theta}: T^{*} \mathcal{T}(S) \longrightarrow T^{*} \mathcal{T}(S)
$$

be the diffeomorphism defined by $(X, \eta) \longmapsto(X, \eta+\theta(X))$, where $\theta$ is the $(1,0)$-form in (3.3). It is easy to see that

$$
T_{B} \circ F_{\theta}=T_{e},
$$

where $T_{B}$ (respectively, $T_{e}$ ) is the diffeomorphism in (2.10) (respectively, $(3.2)$ ), and $F_{\theta}$ is constructed in (3.6).

From (3.7), we have

$$
T_{e}^{*} \Omega_{P}=F_{\theta}^{*}\left(T_{B}^{*} \Omega_{P}\right) .
$$

Therefore, in view of (2.10), we now conclude that (3.4) holds if and only if

$$
F_{\theta}^{*} \Omega_{T}=\Omega_{T} .
$$

On the other hand, from the definition of $F_{\theta}$ it follows that

$$
F_{\theta}^{*} \Omega_{T}-\Omega_{T}=p^{*} d \theta,
$$

where $p$ is the natural projection from $T^{*} \mathcal{T}(S)$ to $\mathcal{T}(S)$. To prove (3.8), we recall that the canonical symplectic form on the total space $T^{*} M$ of the cotangent bundle of a $C^{\infty}$ manifold $M$ is the exterior derivative of a tautological one-form $\alpha_{M}$ on $T^{*} M$. For any smooth one-form $\mu$ on $M$, the diffeomorphism

$$
D_{\mu}: T^{*} M \longrightarrow T^{*} M
$$

defined by $(x, \omega) \longmapsto(x, \omega+\mu(x))$ has the property that

$$
D_{\mu}^{*} \alpha_{M}=\alpha_{M}+q^{*} \mu,
$$

where $q: T^{*} M \longrightarrow M$ is the natural projection. The identity (3.8) follows immediately from this fact. 
Since $p$ in (3.8) is a submersion, the two-form $p^{*} d \theta$ vanishes if and only if $d \theta$ vanishes. Consequently, using (3.8) we now conclude that (3.4) holds if and only if (3.5) holds.

To prove that (3.5) holds, we first note that since both $B$ and $e$ are holomorphic sections of the projection $\varphi$ in $(2.6)$, the $(1,0)$-form $\theta$ in (3.3) is holomorphic. Hence $d \theta$ is a $(2,0)$-form, or in other words,

$$
d \theta \in C^{\infty}\left(\mathcal{T}(S), \Omega_{\mathcal{T}(S)}^{2,0}\right) .
$$

Let

$$
\phi: \mathcal{T}(S) \longrightarrow \mathcal{P}(S)
$$

be the smooth section given by the Fuchsian uniformization. Let

$$
\alpha:=e-\phi \in C^{\infty}\left(\mathcal{T}(S), T^{*} \mathcal{T}(S)\right)
$$

and

$$
\beta:=B-\phi \in C^{\infty}\left(\mathcal{T}(S), T^{*} \mathcal{T}(S)\right)
$$

be the smooth $(1,0)$-forms on $\mathcal{T}(S)$, where $\phi, e$ and $B$ are the sections in (3.10), (3.1) and (2.9), respectively.

From a theorem due to McMullen, [9, p. 350, Theorem 7.1], we have that

$$
d \beta \in C^{\infty}\left(\mathcal{T}(S), \Omega_{\mathcal{T}(S)}^{1,1}\right)
$$

(in fact, $d \beta=\sqrt{-1} \cdot \omega_{\mathrm{WP}}$, where $\omega_{\mathrm{WP}}$ is the Weil-Petersson symplectic form on $\mathcal{T}(S)$ ). Moreover, Theorem 9.2 in $[\mathbf{9}$, p. 355] states that

$$
d \alpha=d \beta .
$$

We note that in $[\mathbf{9}$, Theorem 9.2], this statement is proved for the Schottky uniformization. However, the proof remains unchanged for any smooth section $f$ (as in (2.7)) as long as $f$ is holomorphic and Theorem 9.1 of $[\mathbf{9}$, p. 355] applies to it ${ }^{1}$. Both the sections $e$ and $B$ clearly satisfy these two conditions. (We also note that $[\mathbf{9}$, Theorem 9.2] gives an alternative proof of a theorem of Takhtazan and Zograf in [10].)

We note that $\theta$ in (3.3) satisfies the identity

$$
\theta=\alpha-\beta,
$$

where $\alpha$ and $\beta$ are constructed in (3.11) and (3.12), respectively. Therefore, from (3.13) and (3.14), we have

$$
d \theta \in C^{\infty}\left(\mathcal{T}(S), \Omega_{\mathcal{T}(S)}^{1,1}\right) .
$$

Comparing this with (3.9) we now conclude that

$$
d \theta=0 .
$$

\footnotetext{
${ }^{1}$ We thank Curtis T. McMullen for clarifying this.
} 
As we observed earlier, this implies that (3.4) holds. This completes the proof of the theorem.

Remark 3.2. Take any section $f: \mathcal{T}(S) \longrightarrow \mathcal{P}(S)$ as in (2.7) such that

1) $f$ is holomorphic, and

2) Theorem 9.1 of $[\mathbf{9}$, p. 355] applies to $f$.

Consider the biholomorphism $T_{f}$ constructed in (2.8). The proof of Theorem 3.1 gives

$$
T_{f}^{*} \Omega_{P}=\pi \cdot \Omega_{T}
$$

Apart from the section $\mathcal{T}(S) \longrightarrow \mathcal{P}(S)$ given by the Earle uniformization, the section given by the Schottky uniformization also satisfies the two conditions stated above.

\section{References}

[1] L. Bers, Fiber spaces over Teichmüller spaces, Acta Math. 130 (1973), 89-126.

[2] I. Biswas and A.K. Raina, Projective structures on a Riemann surface, II, Int. Math. Res. Not. 13 (1999), 685-716.

[3] C.J. Earle, Some intrinsic coordinates on Teichmüller space, Proc. Amer. Math. Soc. 83 (1981), 527-531.

[4] W.M. Goldman, The symplectic nature of fundamental group of surfaces, Adv. Math. 54 (1984), 200-225.

[5] R.C. Gunning, Lectures on Riemann surfaces, Princeton Mathematical Notes 2, Princeton University Press, Princeton, NJ, 1966.

[6] D.A. Hejhal, Monodromy groups and linearly polymorphic functions, Acta Math. 135 (1975), 1-55.

[7] J.H. Hubbard, The monodromy of projective structures, in 'Riemann surfaces and related topics' (I. Kra and B. Maskit, eds.), Proceedings of the 1978 Stony Brook Conference, Princeton University Press, Princeton, NJ, 1981, 257-275.

[8] S. Kawai, The symplectic nature of the space of projective connections on Riemann surfaces, Math. Ann. 305 (1996), 161-182.

[9] C.T. McMullen, The moduli space of Riemann surfaces is Kähler hyperbolic, Ann. of Math. 151 (2000), 327-357.

[10] P.G. Zograf and L.A. Takhtadzhyan, On uniformization of Riemann surfaces and the Weil-Petersson metric on Teichmüller and Schottky spaces, Math. USSR Sbornik 60 (1988), 297-313.

School of Mathematics

Tata Institute of Fundamental Research

HOMI BHABHA ROAD

BOMBAY 400005, InDiA

E-mail address: pablo@math.tifr.res.in

indranil@math.tifr.res.in

Received 9/24/2007, accepted 1/17/2008 